\title{
Malignant Fibrous Histiocytoma
}

Malignant fibrous histiocytoma (MFH) is currently considered an obsolete term used in the past for tumors now classified mainly as undifferentiated pleomorphic sarcoma ( $\rightarrow$ see dedicated section). 